\title{
An Opposition-Based Group Search Optimizer with Diversity Guidance
}

\author{
Dan Wang, Congcong Xiong, and Xiankun Zhang \\ School of Computer Science and Information Engineering, Tianjin University of Science \& Technology, Tianjin 300222, China \\ Correspondence should be addressed to Dan Wang; wanghzc@163.com
}

Received 13 July 2015; Revised 1 November 2015; Accepted 8 November 2015

Academic Editor: Miguel A. Salido

Copyright ( 2015 Dan Wang et al. This is an open access article distributed under the Creative Commons Attribution License, which permits unrestricted use, distribution, and reproduction in any medium, provided the original work is properly cited.

\begin{abstract}
Group search optimizer (GSO) which is an effective evolutionary algorithm has been successfully applied in many applications. However, the purely stochastic resampling or selection mechanism in GSO leads to long computing time and premature convergence. In this paper, we propose a diversity-guided group search optimizer (DGSO) with opposition-based learning (OBL) to overcome these limitations. Opposition-based learning is utilized to accelerate the convergence rate of GSO, while the diversity guidance (DG) is used to increase the diversity of population. When compared with the standard GSO, a novel operator using OBL and DG is developed for the population initialization as well as the generation jumping. A comprehensive set of 19 complex benchmark functions is used for experiment verification and is compared to the original GSO algorithm. Numerical experiments show that the proposed DGSO leads to better performance in comparison with the standard GSO in the convergence rate and the solution accuracy.
\end{abstract}

\section{Introduction}

During the past few decades, evolutionary algorithms (EAs) have been developed rapidly and even improved the social development increasingly [1-3]. Diversity of EAs such as Genetic Algorithm (GA) [4], Differential Evolutionary (DE) algorithm [5-7], Ant Colony Optimization (ACO) [8, 9], and Particle Swarm Optimizer (PSO) $[10,11]$ have been proposed, and they also have been successfully applied in lots of applications in practice. It is noted that most of EAs are developed based on the swarm intelligence coming from the nature world.

Along with this viewpoint, a novel swarm intelligence algorithm, namely, group search optimizer (GSO), inspired by animal behavior [12], has been proven to get more competitive performance in comparison with some typical swarm intelligence algorithms such as PSO when dealing with some complex optimization problems [13]. The GSO algorithm is composed by one initialization operator and three evolutionary operators. Like the other EAs, the initialization operator of GSO is realized based on purely random searching. In spite of the guidance of animal behavior mechanism, the three operators are also based on random searching to some extent. It is evident that sometimes such random searching mechanism does not come with the optimal solution in the given time. In other words, the defect of the random mechanism leads to long computing time and premature convergence [14-16]. On the one hand, the GSO algorithm is a population-based optimization algorithm, which is prone to suffer from long computing times. On the other hand, random initialization and selection arrives at the best optimization solution only in good luck without any prior information. These two limitations prevent the development of the GSO and restrict the application of GSO in practice.

In this paper, we propose a new evolutionary algorithm, namely, diversity-guided group search optimizer (DGSO), to alleviate the two drawbacks of GSO. The proposed DGSO is realized based on diversity guidance (DG) and a socalled opposition-based learning (OBL) originally coming from neural networks field [17-20]. Diversity guidance is used to increase the diversity of the population of GSO, while opposition-based learning is utilized to accelerate the 
Line\# Procedure of GSO algorithm
(1) Begin
(2) Initialization
(3) Evaluate the fitness of the current member;
(4) Find a producer;
(5) Producing
(6) Randomly choose three points based on (1);
(7) Search the best resource, otherwise stay the current position based on (2);
(8) If the producer can't search a better area, then change the angle by using (3);
(9) Scrounging
(10) Randomly choose some group members as the scrounger
(11) Dispersion
(12) Generate a head angle using (2)
(13) Obtain a random distance based on (4) and then move to a new point using (5)
(14) Search a better solution, report the final optimal solution
(15) End

Algorithm 1: Procedure of the GSO algorithm.

convergence speed. Numerical experiments are carried out on several benchmark functions to evaluate the performance of the proposed DGSO.

The structure of the paper is arranged as follows. Section 2 recalls the GSO algorithm. Section 3 presents diversityguided group search optimizer (DGSO) with oppositionbased learning. Section 4 reports the experimental results. At last, some conclusions are drawn in Section 5.

\section{Group Search Optimizer Algorithm}

Let us recall the original GSO. In the GSO, there are mainly three evolutionary operators: producing, scrounging, and dispersion. The detailed procedure of the GSO is described in Algorithm 1, where $n$ is the dimension; $X_{i}^{k}$ is in the $i$ th member at the $k$ th iteration; $\varphi_{i}^{k}$ is a head angle; $D_{i}^{k}$ is a unit vector [13].

In the producing operator, the producer scans three points through the following expression [19]:

$$
\begin{aligned}
& X_{z}=X_{p}^{k}+r_{1} l_{\max } D_{p}^{k}\left(\varphi^{k}\right), \\
& X_{r}=X_{p}^{k}+r_{1} l_{\max } D_{p}^{k}\left(\varphi^{k}+\frac{r_{2} \theta_{\max }}{2}\right), \\
& X_{l}=X_{p}^{k}+r_{1} l_{\max } D_{p}^{k}\left(\varphi^{k}-\frac{r_{2} \theta_{\max }}{2}\right),
\end{aligned}
$$

where $\theta_{\max }$ is the maximum search angle; $l_{\max }$ is the maximum pursuit distance; $r_{1}$ and $r_{2}$ are parameters generated under the normally distribution; and $r_{2}$ is also a real number in the interval $[0,1]$.

If the current point changed, the angle is

$$
\varphi^{k+1}=\varphi^{k}+r_{2} \alpha_{\max }
$$

where $\alpha_{\max }$ stands for the maximum turning angle [12].
Otherwise the angle is fixed, and the angle is

$$
\varphi^{k+a}=\varphi^{k}
$$

where $a$ is a constant.

In the scrounging operator, we select $80 \%$ candidates from the remainder of population.

In the dispersion operator, we used the random walks [22-25]. And the random distance is

$$
l_{i}=a \cdot r_{1} l_{\max }
$$

The new point is expressed:

$$
X_{i}^{k+1}=X_{i}^{k}+l_{i} D_{i}^{k}\left(\varphi^{k+1}\right) .
$$

\section{Diversity-Guided Group Search Optimizer (DGSO) with Opposition-Based Learning}

As one of the evolutionary algorithms, GSO is the populationbased algorithm, which has the common default; that is, the individuals are selected from a given population randomly generated. To resolve this problem, we used a priori information such as the opposition-based learning, which is applied for making the opposition individuals, and diversity-guided strategy, which is used for improving the diversity of the original GSO. More details of the DGSO are summarized as shown in Algorithm 2.

3.1. Opposition-Based Search Operator. To speed up the convergence of the DGSO, we use a method of opposition-based learning (OBL) $[17,18,26]$. The essence of $\mathrm{OBL}$ is using opposite numbers, which would accelerate the convergence of the DGSO and prevent DGSO from suffering expensive computational times which always happens as one weakness in evolutionary algorithm. Let us memorize the basic concepts.

Opposition-based point is as follows [24]: assume $P=$ $\left(X_{1}, \ldots, X_{n}\right)$ is a point, and the dimension is in $n$, where $X_{i}$ 


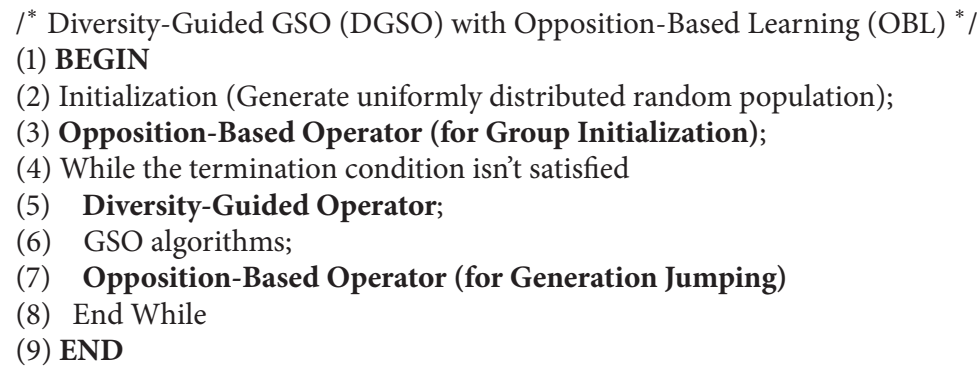

Algorithm 2: Procedure of diversity-guided GSO (DGSO) with opposition-based learning (OBL).

Line\# Procedure of opposition-based learning operator

(1) Begin

(2) Generate the population $P$

(3) for (int $i=0 ; i<\mathrm{NP} ; i++$ )

(4) for (int $j=0 ; j<n ; j++)$

(5) $\mathrm{OP}_{i, j}=a_{j}+b_{j}-P_{i, j}$;

(6) Calculate the fitness value of the $\mathrm{OP}_{i, j}$

(7) Output the some optimal candidates from $\{P, O P\}$;

(8) End

Algorithm 3: Procedure of opposition-based learning operator.

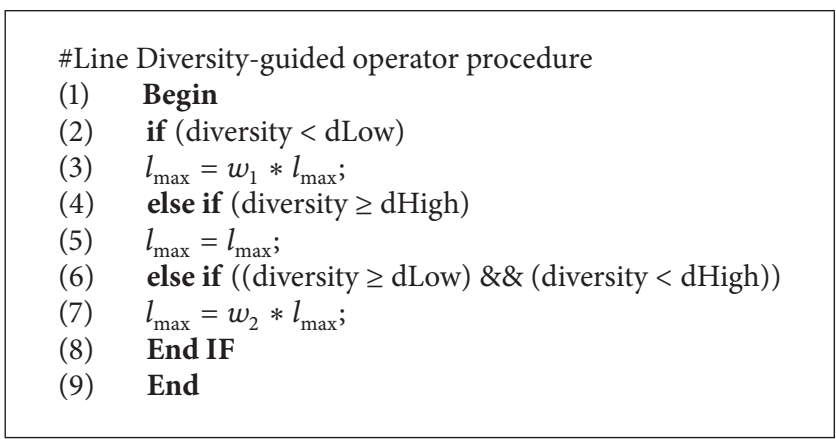

Algorithm 4: Procedure of diversity-guided operator.

is a real number, $X_{i} \in\left[a_{i}, b_{i}\right]$. The opposite point of $P$ is $\widetilde{P}=\left(\widetilde{X_{1}}, \ldots, \widetilde{X_{n}}\right)$, where $\widetilde{X}_{i}=a_{i}+b_{i}-X_{i}$.

Opposition-based optimization (OBL) is as follows [26]: we assume $f(\cdot)$ is the fitness function which is to measure the candidate's fitness value for finding the best candidate solution. According to the above definition, if $f(\widetilde{P}) \geq f(P)$, then the point $P$ replaces $\widetilde{P}$; otherwise the point is fixed and does not change. Hence, the point $P$ and its opposite point $\widetilde{P}$ are evaluated simultaneously to continue the fitter point.

Based on the upper concepts, we embedded the opposition-based operators in the DGSO's initialized group and generation jumping. Algorithm 3 described the procedure of opposition-based learning operator, where $P$ is the population, $O P_{i, j}$ is the opposite point according to $P_{i, j}$, $\mathrm{NP}$ is the population size, and $a_{j}=\min \left(x_{j}^{k}\right), b_{j}=\max \left(x_{j}^{k}\right)$.

3.2. Diversity-Guided Operator. To alleviate the problem of premature convergence, we use a mechanism of diversity guidance as shown in Algorithm 4. Here the diversity of group (population) is computed by the following formula [27]:

$$
\text { diversity }=\frac{1}{|S| \cdot|L|} \cdot \sum_{i=1}^{|S|} \sqrt{\sum_{j=1}^{N}\left(p_{i j}-\bar{p}_{j}\right)^{2}}
$$


TABLE 1: Test functions $f_{1}(x)-f_{11}(x)$.

\begin{tabular}{|c|c|c|c|}
\hline Function & Definition & Domain & Fitness optimum \\
\hline$f_{1}(x)$ & 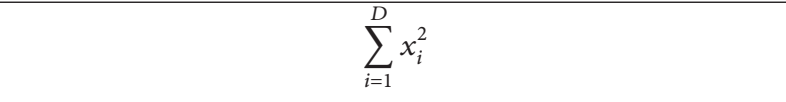 & {$[-100,100]^{D}$} & 0 \\
\hline$f_{2}(x)$ & $\max _{i}\left\{\left|x_{i}\right|, 1 \leq i \leq D\right\}$ & {$[-100,100]^{D}$} & 0 \\
\hline$f_{3}(x)$ & $\sum_{i=1}^{D}\left(100\left(x_{i}^{2}+x_{i+1}\right)^{2}+\left(x_{i}-1\right)^{2}\right)$ & {$[-100,100]^{D}$} & 0 \\
\hline$f_{4}(x)$ & $\sum_{i=1}^{D}\left(x_{i}^{2}-10 \cos \left(2 \pi x_{i}\right)+10\right)$ & {$[-5,5]^{D}$} & 0 \\
\hline$f_{5}(x)$ & $\sum_{i=1}^{D} \frac{x_{i}^{2}}{4000}-\prod_{i=1}^{D} \cos \left(\frac{x_{i}}{\sqrt{i}}\right)+1$ & {$[-600,600]^{D}$} & 0 \\
\hline$f_{6}(x)$ & $-20 \exp \left(-0.2 \sqrt{\frac{1}{D}} \sum_{i=1}^{D} x_{i}^{2}\right)-\exp \left(\frac{1}{D} \sum_{i=1}^{D} \cos \left(2 \pi x_{i}\right)\right)+20+e$ & {$[-32,32]^{D}$} & 0 \\
\hline$f_{7}(x)$ & $\sum_{i=1}\left|x_{i}\right|+\prod_{i=1}\left|x_{i}\right|$ & {$[-10,10]^{D}$} & 0 \\
\hline$f_{8}(x)$ & $\sum_{i=1}\left(\sum_{j=1} x_{j}\right)$ & {$[-65.536,65.536]^{D}$} & 0 \\
\hline$f_{9}(x)$ & $\begin{array}{c}\left(\sum_{i=1} f_{100}\left(x_{i}, x_{i+1}\right)\right)+f_{100}\left(x_{D}, x_{1}\right) \\
f_{100}=\left(x^{2}+y^{2}\right)^{0.25} \cdot\left(\sin ^{2}\left(50 \cdot\left(x^{2}+y^{2}\right)^{0.1}\right)+1\right)\end{array}$ & {$[-100,100]^{D}$} & 0 \\
\hline$f_{10}(x)$ & $\sum_{i=1}^{D}\left(x_{i}^{2}+2 x_{i+1}^{2}-0.3 \cos \left(3 \pi x_{i}\right)-0.4 \cos \left(4 \pi x_{i+1}\right)+0.7\right)$ & {$[-15,15]^{D}$} & 0 \\
\hline$f_{11}(x)$ & $\sum_{i=1}^{D-1}\left(x_{i}^{2}+x_{i+1}^{2}\right)^{0.25}\left(\sin ^{2}\left(50 \cdot\left(x_{i}^{2}+x_{i+1}^{2}\right)^{0.1}\right)+1\right)$ & {$[-100,100]^{D}$} & 0 \\
\hline
\end{tabular}

where $|S|$ is the group size, $|L|$ is the length of the longest diagonal in the search space [26], $N$ is the dimension, $p_{i j}$ is the $j$ th value in the $i$ th member (individual), $\bar{p}$ represents the average point, and $\bar{p}_{j}$ stands for the $j$ th value of $\bar{p}$. Algorithm 4 described the procedure of diversity-based operator, where $l_{\max }$ is a given number used in formula (1); $w_{1}, w_{2}$ are set as 0.33 and 0.66 , individually.

\section{Experimental Studies}

In this section, we demonstrate the detailed experimental studies of the proposed DGSO. First, we describe test functions and many experimental settings. Second, we compared the DGSO to other algorithms using four comparative analyses, mean and standard deviation, convergence speed, contribution of diversity-guided operator, and contribution of opposite points.

\subsection{Experimental Settings}

(1) Test Functions. We select 19 classical benchmark functions described in [28], where the functions $f_{1}(x)-f_{6}(x)$ are selected from CEC 2008 Special Session [29], the functions $f_{7}(x)-f_{11}(x)$ were provided from ISDA 2009 Workshop [30], and the functions $f_{12}(x)-f_{19}(x)$ are the hybrid functions based on the previous functions $f_{1}(x)-f_{11}(x)$ [28]. Test functions $f_{1}(x)-f_{11}(x)$ are shown in Table 1; test functions $f_{12}(x)-f_{19}(x)$ are shown in Table 2. In the experimental process, we set the population size to 100 . For each algorithm, we run 25 times. In the problem size $D$, we set $D \in$ $\{50,100,200\}$. According to [31], MAX_FES is set to $5000 * D$. All of the algorithms are terminated when the MAX_FES is reached.

(2) Experimental Settings. We used five different algorithms for comparing: GSO [13], RGSO, OGSO, RDGSO, and DGSO. All of them are based on the GSO algorithm. The only difference is whether they are applied in the manner of initialization and evolutionary operators. More details are described in Table 3. Through all experiments, we set the head angle $\varphi_{i}^{k}$ to be $\pi / 4$; the maximum search angle $\theta_{\max }$ is $\pi / a^{2}$, where the value of $a$ is a given number and $\theta_{\max } / 2$ is the maximum turning angle (see [13]).

4.2. Experimental Results. In this section, we report and analyze the results of the proposed algorithm with some popular algorithms reported in the literatures. 
TABle 2: Test functions $f_{12}(x)-f_{19}(x)$.

\begin{tabular}{|c|c|c|c|c|c|}
\hline & & Function & $m_{n s}$ & Domain & $\mathrm{FO}$ \\
\hline$f_{12}(x)=f_{9}(x) \oplus f_{1}(x)$ & $\begin{array}{l}f_{9}(x) \\
f_{1}(x)\end{array}$ & $\begin{array}{c}\left(\sum_{i=1}^{D-1} f_{100}\left(x_{i}, x_{i+1}\right)\right)+f_{100}\left(x_{D}, x_{1}\right) \\
f_{100}=\left(x^{2}+y^{2}\right)^{0.25} \cdot\left(\sin ^{2}\left(50 \cdot\left(x^{2}+y^{2}\right)^{0.1}\right)+1\right) \\
\sum_{i=1}^{D} x_{i}^{2}\end{array}$ & 0.25 & {$[-100,100]^{D}$} & 0 \\
\hline$f_{13}(x)=f_{9}(x) \oplus f_{3}(x)$ & $\begin{array}{l}f_{9}(x) \\
f_{3}(x)\end{array}$ & $\begin{array}{c}\left(\sum_{i=1}^{D-1} f_{100}\left(x_{i}, x_{i+1}\right)\right)+f_{100}\left(x_{D}, x_{1}\right) \\
f_{100}=\left(x^{2}+y^{2}\right)^{0.25} \cdot\left(\sin ^{2}\left(50 \cdot\left(x^{2}+y^{2}\right)^{0.1}\right)+1\right) \\
\sum_{i=1}^{D}\left(100\left(x_{i}^{2}+x_{i+1}\right)^{2}+\left(x_{i}-1\right)^{2}\right)\end{array}$ & 0.25 & {$[-100,100]^{D}$} & 0 \\
\hline$f_{14}(x)=f_{9}(x) \oplus f_{4}(x)$ & $\begin{array}{l}f_{9}(x) \\
f_{4}(x)\end{array}$ & $\begin{array}{c}\left(\sum_{i=1}^{D-1} f_{100}\left(x_{i}, x_{i+1}\right)\right)+f_{100}\left(x_{D}, x_{1}\right) \\
f_{100}=\left(x^{2}+y^{2}\right)^{0.25} \cdot\left(\sin ^{2}\left(50 \cdot\left(x^{2}+y^{2}\right)^{0.1}\right)+1\right) \\
\sum_{i=1}^{D}\left(x_{i}^{2}-10 \cos \left(2 \pi x_{i}\right)+10\right)\end{array}$ & 0.25 & {$[-5,5]^{D}$} & 0 \\
\hline$f_{15}(x)=f_{10}(x) \oplus f_{7}(x)$ & $\begin{array}{l}f_{10}(x) \\
f_{7}(x)\end{array}$ & $\begin{array}{c}\sum_{i=1}^{D}\left(x_{i}^{2}+2 x_{i+1}^{2}-0.3 \cos \left(3 \pi x_{i}\right)-0.4 \cos \left(4 \pi x_{i+1}\right)+0.7\right) \\
\sum_{i=1}^{D}\left|x_{i}\right|+\prod_{i=1}^{D}\left|x_{i}\right|\end{array}$ & 0.25 & {$[-10,10]^{D}$} & 0 \\
\hline$f_{16}(x)=f_{9}(x) \oplus f_{1}(x)$ & $\begin{array}{l}f_{9}(x) \\
f_{1}(x)\end{array}$ & $\begin{array}{c}\left(\sum_{i=1}^{D-1} f_{100}\left(x_{i}, x_{i+1}\right)\right)+f_{100}\left(x_{D}, x_{1}\right) \\
f_{100}=\left(x^{2}+y^{2}\right)^{0.25} \cdot\left(\sin ^{2}\left(50 \cdot\left(x^{2}+y^{2}\right)^{0.1}\right)+1\right) \\
\sum_{i=1}^{D} x_{i}^{2}\end{array}$ & 0.5 & {$[-100,100]^{D}$} & 0 \\
\hline$f_{17}(x)=f_{9}(x) \oplus f_{3}(x)$ & $\begin{array}{l}f_{9}(x) \\
f_{3}(x)\end{array}$ & $\begin{array}{c}\left(\sum_{i=1}^{D-1} f_{100}\left(x_{i}, x_{i+1}\right)\right)+f_{100}\left(x_{D}, x_{1}\right) \\
f_{100}=\left(x^{2}+y^{2}\right)^{0.25} \cdot\left(\sin ^{2}\left(50 \cdot\left(x^{2}+y^{2}\right)^{0.1}\right)+1\right) \\
\sum^{D}\left(100\left(x_{i}^{2}+x_{i+1}\right)^{2}+\left(x_{i}-1\right)^{2}\right)\end{array}$ & 0.75 & {$[-100,100]^{D}$} & 0 \\
\hline$f_{18}(x)=f_{9}(x) \oplus f_{4}(x)$ & $f_{9}(x)$ & $\begin{array}{c}\left(\sum_{i=1}^{D-1} f_{100}\left(x_{i}, x_{i+1}\right)\right)+f_{100}\left(x_{D}, x_{1}\right) \\
f_{100}=\left(x^{2}+y^{2}\right)^{0.25} \cdot\left(\sin ^{2}\left(50 \cdot\left(x^{2}+y^{2}\right)^{0.1}\right)+1\right) \\
\sum_{i=1}^{D}\left(x_{i}^{2}-10 \cos \left(2 \pi x_{i}\right)+10\right)\end{array}$ & 0.75 & {$[-5,5]^{D}$} & 0 \\
\hline$f_{19}(x)=f_{10}(x) \oplus f_{7}(x)$ & $\begin{array}{l}f_{10}(x) \\
f_{7}(x)\end{array}$ & $\begin{array}{c}\sum_{i=1}^{D}\left(x_{i}^{2}+2 x_{i+1}^{2}-0.3 \cos \left(3 \pi x_{i}\right)-0.4 \cos \left(4 \pi x_{i+1}\right)+0.7\right) \\
\sum_{i=1}^{D}\left|x_{i}\right|+\prod_{i=1}^{D}\left|x_{i}\right|\end{array}$ & 0.75 & {$[-10,10]^{D}$} & 0 \\
\hline
\end{tabular}

${ }^{*} \mathrm{FO}$ denotes fitness optimum.

(1) Mean and Standard Deviation. Two performance indexes, mean and standard deviation, are utilized to measure the performance of different algorithms whose strategies are described in Table 3. Table 4 provides the average values when $D$ is set as 30 . As shown in Table 4, the proposed algorithm DGSO performs better than the other algorithms (GSO, OGSO, and RDGSO) in most benchmark functions.
Table 5 summarizes the comparison of DGSO, GSO, and RDGSO with the different dimensions $(D=50,100,200)$, respectively. It is evident that DGSO performs better than the other reported algorithms especially in case of the high dimension functions.

(2) Convergence Speed. Next we compare the convergence speed between the DGSO and the other four algorithms 
TABLE 3: Strategies used in the five GSO algorithms.

\begin{tabular}{|c|c|c|}
\hline Algorithms & Manner of initialization & $\begin{array}{c}\text { Manner of } \\
\text { evolutionary } \\
\text { operators }\end{array}$ \\
\hline GSO & Random & Random \\
\hline RGSO & Random & $\begin{array}{c}\text { Opposition-based } \\
\text { learning }\end{array}$ \\
\hline OGSO & Opposition-based learning & $\begin{array}{c}\text { Opposition-based } \\
\text { learning }\end{array}$ \\
\hline RDGSO & Random & $\begin{array}{c}\text { Opposition-based } \\
\text { learning and } \\
\text { diversity-guided } \\
\text { operator }\end{array}$ \\
\hline DGSO & Opposition-based learning & $\begin{array}{l}\text { Opposition-based } \\
\text { learning and } \\
\text { diversity-guided } \\
\text { operator }\end{array}$ \\
\hline
\end{tabular}

TABLE 4: Average fitness values tested on benchmark functions $(D=30)$.

\begin{tabular}{|c|c|c|c|c|c|c|c|c|}
\hline \multirow{2}{*}{${ }^{*} \mathrm{~F}$} & \multicolumn{2}{|c|}{ GSO } & \multicolumn{2}{|c|}{ OGSO } & \multicolumn{2}{|c|}{ RDGSO } & \multicolumn{2}{|c|}{ DGSO } \\
\hline & Mean & Std. & Mean & Std. & Mean & Std. & Mean & Std. \\
\hline $\mathrm{F} 1$ & $0.00 \mathrm{E}+00$ & $0.00 \mathrm{E}+00$ & $0.00 \mathrm{E}+00$ & $0.00 \mathrm{E}+00$ & $0.00 \mathrm{E}+00$ & $0.00 \mathrm{E}+00$ & $0.00 \mathrm{E}+00$ & $0.00 \mathrm{E}+00$ \\
\hline F2 & $0.00 \mathrm{E}+00$ & $0.00 \mathrm{E}+00$ & $1.43 E-05$ & $1.24 E-05$ & $0.00 \mathrm{E}+00$ & $0.00 \mathrm{E}+00$ & $2.14 E-03$ & $2.03 E-04$ \\
\hline F3 & $7.99 E+01$ & $5.25 E-01$ & $6.97 E+02$ & $2.86 E+01$ & $6.56 E+01$ & $6.03 E-01$ & $4.55 E+01$ & $3.94 E+01$ \\
\hline F4 & $0.00 \mathrm{E}+00$ & $0.00 \mathrm{E}+00$ & $0.00 \mathrm{E}+00$ & $0.00 \mathrm{E}+00$ & $0.00 \mathrm{E}+00$ & $0.00 \mathrm{E}+00$ & $0.00 E+00$ & $0.00 \mathrm{E}+00$ \\
\hline F5 & $0.00 \mathrm{E}+00$ & $0.00 \mathrm{E}+00$ & $0.00 \mathrm{E}+00$ & $0.00 \mathrm{E}+00$ & $0.00 \mathrm{E}+00$ & $0.00 \mathrm{E}+00$ & $0.00 \mathrm{E}+00$ & $0.00 \mathrm{E}+00$ \\
\hline F6 & $0.00 \mathrm{E}+00$ & $0.00 \mathrm{E}+00$ & $0.00 E+00$ & $0.00 \mathrm{E}+00$ & $0.00 \mathrm{E}+00$ & $0.00 E+00$ & $0.00 \mathrm{E}+00$ & $0.00 \mathrm{E}+00$ \\
\hline F7 & $0.00 \mathrm{E}+00$ & $0.00 \mathrm{E}+00$ & $0.00 \mathrm{E}+00$ & $0.00 \mathrm{E}+00$ & $0.00 \mathrm{E}+00$ & $0.00 \mathrm{E}+00$ & $0.00 E+00$ & $0.00 \mathrm{E}+00$ \\
\hline F8 & $0.00 \mathrm{E}+00$ & $0.00 \mathrm{E}+00$ & $0.00 \mathrm{E}+00$ & $0.00 \mathrm{E}+00$ & $0.00 \mathrm{E}+00$ & $0.00 \mathrm{E}+00$ & $0.00 \mathrm{E}+00$ & $0.00 \mathrm{E}+00$ \\
\hline F9 & $0.00 \mathrm{E}+00$ & $0.00 \mathrm{E}+00$ & $0.00 \mathrm{E}+00$ & $0.00 \mathrm{E}+00$ & $0.00 \mathrm{E}+00$ & $0.00 \mathrm{E}+00$ & $0.00 E+00$ & $0.00 \mathrm{E}+00$ \\
\hline F10 & $0.00 \mathrm{E}+00$ & $0.00 \mathrm{E}+00$ & $0.00 \mathrm{E}+00$ & $0.00 \mathrm{E}+00$ & $0.00 \mathrm{E}+00$ & $0.00 \mathrm{E}+00$ & $0.00 \mathrm{E}+00$ & $0.00 \mathrm{E}+00$ \\
\hline F11 & $0.00 \mathrm{E}+00$ & $0.00 \mathrm{E}+00$ & $0.00 \mathrm{E}+00$ & $0.00 \mathrm{E}+00$ & $0.00 \mathrm{E}+00$ & $0.00 \mathrm{E}+00$ & $0.00 \mathrm{E}+00$ & $0.00 \mathrm{E}+00$ \\
\hline F12 & $0.00 \mathrm{E}+00$ & $0.00 \mathrm{E}+00$ & $0.00 \mathrm{E}+00$ & $0.00 \mathrm{E}+00$ & $0.00 \mathrm{E}+00$ & $0.00 \mathrm{E}+00$ & $0.00 E+00$ & $0.00 \mathrm{E}+00$ \\
\hline F13 & $6.43 E+01$ & $7.47 E-01$ & $2.34 E+01$ & $2.46 E+01$ & $3.93 E+01$ & $8.87 E-01$ & $2.87 E+01$ & $2.49 E+01$ \\
\hline F14 & $0.00 \mathrm{E}+00$ & $0.00 \mathrm{E}+00$ & $0.00 \mathrm{E}+00$ & $0.00 \mathrm{E}+00$ & $0.00 \mathrm{E}+00$ & $0.00 \mathrm{E}+00$ & $0.00 E+00$ & $0.00 \mathrm{E}+00$ \\
\hline F15 & $0.00 \mathrm{E}+00$ & $0.00 \mathrm{E}+00$ & $0.00 \mathrm{E}+00$ & $0.00 \mathrm{E}+00$ & $0.00 \mathrm{E}+00$ & $0.00 \mathrm{E}+00$ & $0.00 \mathrm{E}+00$ & $0.00 \mathrm{E}+00$ \\
\hline F16 & $0.00 \mathrm{E}+00$ & $0.00 \mathrm{E}+00$ & $0.00 \mathrm{E}+00$ & $0.00 \mathrm{E}+00$ & $0.00 \mathrm{E}+00$ & $1.39 \mathrm{E}-158$ & $0.00 \mathrm{E}+00$ & $0.00 \mathrm{E}+00$ \\
\hline F17 & $3.07 E+01$ & $8.95 E-01$ & $2.14 E+02$ & $2.40 E+01$ & $1.66 E+01$ & $7.42 E-01$ & $9.38 E+00$ & $1.63 E+01$ \\
\hline F18 & $0.00 \mathrm{E}+00$ & $0.00 \mathrm{E}+00$ & $0.00 \mathrm{E}+00$ & $0.00 \mathrm{E}+00$ & $0.00 \mathrm{E}+00$ & $0.00 \mathrm{E}+00$ & $0.00 \mathrm{E}+00$ & $0.00 \mathrm{E}+00$ \\
\hline F19 & $2.15 E+01$ & $2.98 E-01$ & $3.45 E+01$ & $2.64 E+01$ & $1.14 E-02$ & $4.29 E-03$ & $6.96 E-03$ & $4.98 E-03$ \\
\hline
\end{tabular}

${ }^{*} \mathrm{~F}$ : functions.

including GSO, OGSO, RGSO, and RDGSO. Figure 1 depicts the values of fitness with the increasing of FES [32-34] for $f_{1}-f_{19}$. It shows that the convergence speed of DGSO is faster than the other four algorithms.

(3) Contribution of Diversity-Guided Operator. Now let us analyze the contribution of diversity-guided operator. For convenience, two algorithms DGSO that used the diversityguided operator and OGSO that is the opposition-based algorithm without diversity-guided operator are compared.
The experiment results are summarized in Table 6. It is clear that the group (population) of DGSO obtains more diversity in comparison with OGSO in most of benchmark functions.

(4) Comparison Results of Other Optimization Algorithms. The proposed DGSO is also compared with some other well-known evolutionary algorithms [21] based on these benchmark functions.

Table 7 describes the comparison results of DGSO and some other algorithms when $D=100$. As shown in 
TABLE 5: Average fitness values tested on benchmark functions with increasing dimension.

\begin{tabular}{|c|c|c|c|c|c|c|c|c|c|c|}
\hline \multicolumn{2}{|c|}{ Functions } & \multicolumn{3}{|c|}{ GSO } & \multicolumn{3}{|c|}{ RDGSO } & \multicolumn{3}{|c|}{ DGSO } \\
\hline & & $\mathrm{im}=50$ & 100 & 200 & $\operatorname{dim}=50$ & 100 & 200 & $\operatorname{dim}=50$ & 100 & 200 \\
\hline \multirow[b]{2}{*}{$\Gamma$} & Mean & $0 E+00$ & $4.60 E+00$ & $20 E+00$ & $00 E+00$ & $0.00 \mathrm{E}+00$ & $0.00 \mathrm{E}+00$ & $.00 E+00$ & $0.00 \mathrm{E}+00$ & $00 E+0$ \\
\hline & & $6 E+00$ & $00 \mathrm{E}+00$ & $7 E+00$ & $00 \mathrm{E}+00$ & $0.00 \mathrm{E}+00$ & $0.00 \mathrm{E}+00$ & $.00 \mathrm{E}+00$ & $0.00 \mathrm{E}+00$ & $0.00 \mathrm{E}+00$ \\
\hline \multirow{2}{*}{$\mathrm{F} 2$} & Mean & $0 E+00$ & $.80 E+00$ & $0 E+00$ & $00 \mathrm{E}+00$ & $0.00 \mathrm{E}+00$ & $0.00 \mathrm{E}+00$ & & $5.00 E+00$ & $3.67 E+00$ \\
\hline & Std. & $7 E+00$ & $00 \mathrm{E}+00$ & $3.91 E+00$ & $00 \mathrm{E}+00$ & $0.00 \mathrm{E}+00$ & $0.00 \mathrm{E}+00$ & $00 \mathrm{E}+00$ & $3.61 E+00$ & $.51 E+00$ \\
\hline \multirow{2}{*}{ F3 } & Mean & $50 E+00$ & $3.20 E+00$ & $60 E+00$ & $2.40 E+00$ & $2.33 E+00$ & $2.33 E+00$ & $4.80 E+00$ & $2.33 E+00$ & $4.00 E+00$ \\
\hline & td. & $30 E+00$ & $4.76 E-02$ & $1.52 E+00$ & $3.36 E+00$ & $2.08 E+00$ & $1.53 E+00$ & $00 \mathrm{E}+00$ & $2.52 E+00$ & $2.65 E+00$ \\
\hline \multirow{2}{*}{$\mathrm{F} 4$} & Mean & $10 E+00$ & $5.20 E+00$ & $3.00 E+00$ & $0.00 \mathrm{E}+00$ & $0.00 \mathrm{E}+00$ & $0.00 \mathrm{E}+00$ & $00 \mathrm{E}+00$ & $0.00 \mathrm{E}+00$ & $00 \mathrm{E}+00$ \\
\hline & & & $0.00 \mathrm{E}+00$ & & & $0.00 \mathrm{E}+00$ & & & & $00 \mathrm{E}+00$ \\
\hline \multirow{2}{*}{ F5 } & & & $E+00$ & & & $0.00 E+00$ & & & $0.00 \mathrm{E}+00$ & +00 \\
\hline & & $5 E+00$ & $0.00 \mathrm{E}+00$ & $2.51 E+00$ & $00 \mathrm{E}+00$ & $0.00 \mathrm{E}+00$ & $00 \mathrm{E}+00$ & & $0.00 \mathrm{E}+00$ & $0.00 \mathrm{E}+00$ \\
\hline \multirow{2}{*}{ F6 } & Mean & $0 E+00$ & $2.40 E+00$ & $40 F+00$ & $00 \mathrm{E}+00$ & $0.00 \mathrm{E}+00$ & $0.00 \mathrm{E}+00$ & & $0.00 \mathrm{E}+00$ & $0.00 \mathrm{E}+00$ \\
\hline & & & $0.00 \mathrm{E}+00$ & & $00 \mathrm{E}+00$ & $0.00 \mathrm{E}+00$ & & & $0.00 \mathrm{E}+00$ & $0.00 E+00$ \\
\hline \multirow{2}{*}{ F7 } & & & $00 E+00$ & & & & & & $0.00 \mathrm{E}+00$ & \\
\hline & & & $0 \mathrm{E}+00$ & & & $0.00 \mathrm{E}$ & & & 00 & \\
\hline \multirow{2}{*}{ F8 } & & & $80 E+00$ & & & & & & & \\
\hline & & & $0.00 \mathrm{E}+00$ & & & $0.00 \mathrm{E}$ & & & 00 & 00 \\
\hline \multirow{2}{*}{ F9 } & & & $20 E+00$ & & & & & & -00 & \\
\hline & & & $\mathbf{E}+$ & & & & & & & \\
\hline \multirow{2}{*}{ F10 } & & & $3.40 E+00$ & & & & & & & \\
\hline & & & 0.0 & & & & & & & \\
\hline \multirow{2}{*}{ F11 } & & & & & & & & & & \\
\hline & & & $0.00 \mathrm{E}+00$ & & & & & & $0.00 \mathrm{E}+00$ & \\
\hline & Mean & & $4.40 E+00$ & & & $0.00 \mathrm{E}+00$ & & & $0.00 \mathrm{E}+00$ & \\
\hline & & & $0.00 \mathrm{E}+00$ & & & & & & & \\
\hline \multirow{2}{*}{ F13 } & & & $80 E+00$ & & & & & & & \\
\hline & & & $.23 E-03$ & & & & & & $4.16 E+00$ & \\
\hline \multirow{2}{*}{ F14 } & Mean & & $4.60 E+00$ & & & & & & & \\
\hline & Std. & $E+00$ & $0.00 \mathrm{E}+00$ & $2.83 E+00$ & $0.00 \mathrm{E}+00$ & $0.00 \mathrm{E}+00$ & $0.00 \mathrm{E}+00$ & $0.00 \mathrm{E}+00$ & $0.00 \mathrm{E}+00$ & $.00 \mathrm{E}+00$ \\
\hline \multirow{2}{*}{ F15 } & Mean & $20 E+00$ & $3.60 E+00$ & $4.60 E+00$ & $00 E+00$ & $0.00 \mathrm{E}+00$ & $0.00 E+00$ & $0.00 \mathrm{E}+00$ & $0.00 \mathrm{E}+00$ & $0.00 E+00$ \\
\hline & Std. & $.78 E+00$ & $0.00 \mathrm{E}+00$ & $3.36 E+00$ & $0.00 \mathrm{E}+00$ & $0.00 \mathrm{E}+00$ & $0.00 \mathrm{E}+00$ & $0.00 \mathrm{E}+00$ & $0.00 \mathrm{E}+00$ & $.00 \mathrm{E}+00$ \\
\hline \multirow{2}{*}{ F16 } & Mean & $40 E+00$ & $6.40 E+00$ & $4.60 E+00$ & $0.00 \mathrm{E}+00$ & $0.00 \mathrm{E}+00$ & $0.00 \mathrm{E}+00$ & $0.00 \mathrm{E}+00$ & $0.00 \mathrm{E}+00$ & $0.00 \mathrm{E}+00$ \\
\hline & Std. & $3.58 E+00$ & $0.00 \mathrm{E}+00$ & $2.07 E+00$ & $0.00 \mathrm{E}+00$ & $0.00 \mathrm{E}+00$ & $0.00 \mathrm{E}+00$ & $0.00 \mathrm{E}+00$ & $0.00 \mathrm{E}+00$ & $0.00 E+00$ \\
\hline \multirow{2}{*}{ F17 } & Mean & & $4.80 E+00$ & & & & & & & $4.00 E+00$ \\
\hline & Std. & $4.09 E+00$ & $1.71 E-06$ & $2.97 E+00$ & $2.88 E+00$ & $1.15 E+00$ & & & $2.31 E+00$ & $2.00 E+00$ \\
\hline \multirow{2}{*}{ F18 } & Mean & $3.60 E+00$ & $3.60 E+00$ & $1.60 E+00$ & $0.00 \mathrm{E}+00$ & $0.00 \mathrm{E}+00$ & $0.00 \mathrm{E}+00$ & $0.00 \mathrm{E}+00$ & $0.00 \mathrm{E}+00$ & $0.00 \mathrm{E}+00$ \\
\hline & Std. & & $0.00 \mathrm{E}+00$ & & $0.00 \mathrm{E}+00$ & $0.00 \mathrm{E}+00$ & $0.00 \mathrm{E}+00$ & $0.00 \mathrm{E}+00$ & $0.00 \mathrm{E}+00$ & $0.00 \mathrm{E}+00$ \\
\hline \multirow{2}{*}{ F19 } & & & $5.00 E+00$ & & & & & & $5.33 E+00$ & $3.33 E+00$ \\
\hline & Std. & $2.92 E+00$ & $2.12 E+01$ & $2.30 E+00$ & $3.36 E+00$ & $1.53 E+00$ & $1.73 E+00$ & $1.79 E+00$ & $2.08 E+00$ & $1.53 E+00$ \\
\hline
\end{tabular}

Table 7, the proposed DGSO obtains promising results in most of benchmark functions when compared with the other algorithms.

\section{Conclusion}

In this paper, we proposed a diversity-guided group search optimizer (DGSO) that is realized based on an oppositionbased learning method and a diversity-guided operator mechanism. Experiments are carried out on 19 benchmark functions to demonstrate that the proposed DGSO performs much better results than the other four GSOs including RDGSO, OGSO, RGSO, and GSO.

\section{Conflict of Interests}

The authors declare that there is no conflict of interests regarding the publication of this paper. 
TABLE 6: Diversity values of the OGSO and DGSO.

\begin{tabular}{|c|c|c|c|c|}
\hline \multirow{2}{*}{ Functions } & \multicolumn{2}{|c|}{ OGSO } & \multicolumn{2}{|c|}{ DGSO } \\
\hline & DIV mean & DIV std. & DIV mean & DIV std. \\
\hline $\mathrm{F} 1$ & $1.19 E-02$ & $3.33 E-04$ & $2.36 E-02$ & $8.77 E-03$ \\
\hline $\mathrm{F} 2$ & $1.42 E-02$ & $3.74 E-04$ & $1.198 E-01$ & $1.85 E-01$ \\
\hline F3 & $2.18 E-02$ & $2.00 E-02$ & $9.01 E-02$ & $1.12 E-01$ \\
\hline $\mathrm{F} 4$ & $1.11 E+00$ & $6.88 E-01$ & $1.40 E+00$ & $1.93 E+00$ \\
\hline F5 & $4.23 E-03$ & $3.76 E-03$ & $2.15 E-03$ & $1.15 E-04$ \\
\hline F6 & $1.66 E-01$ & $1.35 E-01$ & $3.89 E-01$ & $6.01 E-01$ \\
\hline F7 & $8.32 E-01$ & $6.65 E-01$ & $1.16 E-01$ & $3.13 E-02$ \\
\hline F8 & $5.76 E-02$ & $2.12 E-02$ & $2.87 E-02$ & $1.26 E-02$ \\
\hline F9 & $1.96 E-02$ & $1.13 E-02$ & $5.40 E-02$ & $7.14 E-02$ \\
\hline F10 & $1.15 E+00$ & $1.12 E+00$ & $1.05 E-01$ & $3.56 E-02$ \\
\hline F11 & $1.53 E-01$ & $1.43 E-01$ & $9.49 E-02$ & $8.01 E-02$ \\
\hline F12 & $1.23 E-01$ & $1.19 E-01$ & $1.37 E-01$ & $2.09 E-01$ \\
\hline F13 & $3.59 E-01$ & $6.22 E-01$ & $5.70 E-02$ & $7.82 E-02$ \\
\hline F14 & $2.12 E-01$ & $2.01 E-01$ & $3.39 E-01$ & $1.10 E-01$ \\
\hline F15 & $5.08 E-01$ & $4.46 E-01$ & $1.95 E-01$ & $1.52 E-01$ \\
\hline F16 & $1.31 E-01$ & $1.01 E-01$ & $4.17 E-02$ & $3.35 E-02$ \\
\hline F17 & $1.92 E-01$ & $1.65 E-01$ & $6.37 E-01$ & $1.10 E+00$ \\
\hline F18 & $4.49 E-02$ & $3.44 E-02$ & $5.97 E-02$ & $6.92 E-02$ \\
\hline F19 & $1.27 E-01$ & $3.98 E-02$ & $9.09 E-02$ & $2.74 E-02$ \\
\hline
\end{tabular}

TABLE 7: Average fitness values tested on benchmark functions.

\begin{tabular}{|c|c|c|c|c|c|c|c|c|}
\hline \multirow{2}{*}{${ }^{*} \mathrm{~F}$} & \multicolumn{2}{|c|}{ DGSO } & \multicolumn{2}{|c|}{ PSO [10] } & \multicolumn{2}{|c|}{$\mathrm{DE}[5]$} & \multicolumn{2}{|c|}{ RGSO [21] } \\
\hline & Mean & Std. & Mean & Std. & Mean & Std. & Mean & Std. \\
\hline $\mathrm{F} 1$ & $0.00 \mathrm{E}+00$ & $0.00 \mathrm{E}+00$ & $4.69 E+05$ & $3.22 E+04$ & $0.00 \mathrm{E}+00$ & $0.00 \mathrm{E}+00$ & $0.00 \mathrm{E}+00$ & $0.00 \mathrm{E}+00$ \\
\hline $\mathrm{F} 2$ & $5.00 E+00$ & $3.61 E+00$ & $7.57 E+01$ & $6.37 E+00$ & $5.21 E+01$ & $4.12 E+00$ & $3.46 E-02$ & $1.02 E-03$ \\
\hline F3 & $2.33 E+00$ & $2.52 E+00$ & $3.26 E+11$ & $2.06 E+10$ & $2.17 E+02$ & $1.15 E+02$ & $5.69 E+01$ & $4.21 E-01$ \\
\hline $\mathrm{F} 4$ & $0.00 \mathrm{E}+00$ & $0.00 \mathrm{E}+00$ & $7.41 E+02$ & $4.96 E+01$ & $6.38 E+02$ & $1.01 E+02$ & $4.3 E-06$ & $2.31 E-05$ \\
\hline F5 & $0.00 \mathrm{E}+00$ & $0.00 \mathrm{E}+00$ & $9.63 E-01$ & $6.15 E-01$ & $0.00 \mathrm{E}+00$ & $0.00 \mathrm{E}+00$ & $4.20 E+00$ & $1.03 E-01$ \\
\hline F6 & $0.00 \mathrm{E}+00$ & $0.00 \mathrm{E}+00$ & $1.96 E+01$ & $1.83 E-01$ & $2.28 E-10$ & $1.33 E-10$ & $3.72 E+00$ & $0.48 E+00$ \\
\hline F7 & $0.00 \mathrm{E}+00$ & $0.00 \mathrm{E}+00$ & $1.02 E+41$ & $2.26 E+41$ & $2.49 E-11$ & $1.23 E-11$ & $4.20 E+00$ & $2.03 E-01$ \\
\hline F8 & $0.00 \mathrm{E}+00$ & $0.00 \mathrm{E}+00$ & $2.60 E+05$ & $6.45 E+04$ & $3.94 E+03$ & $7.69 E+02$ & $4.78 E+00$ & $4.03 E-01$ \\
\hline F9 & $0.00 \mathrm{E}+00$ & $0.00 \mathrm{E}+00$ & $5.84 E+02$ & $4.06 E+01$ & $1.14 E+00$ & $9.30 E-01$ & $5.13 E+00$ & $2.46 E-01$ \\
\hline F10 & $0.00 \mathrm{E}+00$ & $0.00 \mathrm{E}+00$ & $5.35 E+01$ & $3.82 E+00$ & $5.25 E+00$ & $1.29 E+00$ & $3.40 E+00$ & $1.02 E+00$ \\
\hline F11 & $0.00 \mathrm{E}+00$ & $0.00 \mathrm{E}+00$ & $5.77 E+02$ & $2.09 E+01$ & $5.17 E+00$ & $3.32 E+00$ & $0.00 \mathrm{E}+00$ & $0.00 \mathrm{E}+00$ \\
\hline $\mathrm{F} 12$ & $0.00 \mathrm{E}+00$ & $0.00 \mathrm{E}+00$ & $3.05 E+05$ & $2.71 E+04$ & $6.44 E-03$ & $9.60 E-03$ & $0.00 \mathrm{E}+00$ & $0.00 \mathrm{E}+00$ \\
\hline F13 & $3.33 E+00$ & $4.16 E+00$ & $2.00 E+11$ & $6.15 E+10$ & $7.96 E+01$ & $2.45 E+01$ & $4.31 E+01$ & $2.10 E-01$ \\
\hline F14 & $0.00 \mathrm{E}+00$ & $0.00 \mathrm{E}+00$ & $5.35 E+02$ & $5.24 E+01$ & $4.67 E+02$ & $6.71 E+01$ & $3.27 E+00$ & $1.04 E+00$ \\
\hline F15 & $0.00 \mathrm{E}+00$ & $0.00 \mathrm{E}+00$ & $3.79 E+27$ & $6.57 E+27$ & $4.20 E-01$ & $5.75 E-01$ & $5.41 E+00$ & $1.02 E+00$ \\
\hline F16 & $0.00 \mathrm{E}+00$ & $0.00 \mathrm{E}+00$ & $3.41 E+05$ & $1.89 E+04$ & $5.45 E+02$ & $2.32 E-07$ & $7.02 E+00$ & $1.34 E+00$ \\
\hline F17 & $5.33 E+00$ & $2.31 E+00$ & $5.24 E+10$ & $7.13 E+08$ & $3.59 E+10$ & $7.14 E+04$ & $2.43 E+01$ & $3.69 E-01$ \\
\hline F18 & $0.00 \mathrm{E}+00$ & $0.00 \mathrm{E}+00$ & $2.05 E+05$ & $4.85 E+03$ & $8.25 E-02$ & $6.47 E-02$ & $3.21 E+00$ & $2.68 E-01$ \\
\hline F19 & $5.33 E+00$ & $2.08 E+00$ & $2.13 E+10$ & $9.43 E+09$ & $1.93 E+01$ & $1.53 E+00$ & $2.15 E+01$ & $2.98 E-01$ \\
\hline
\end{tabular}

${ }^{*} \mathrm{~F}$ : functions. 

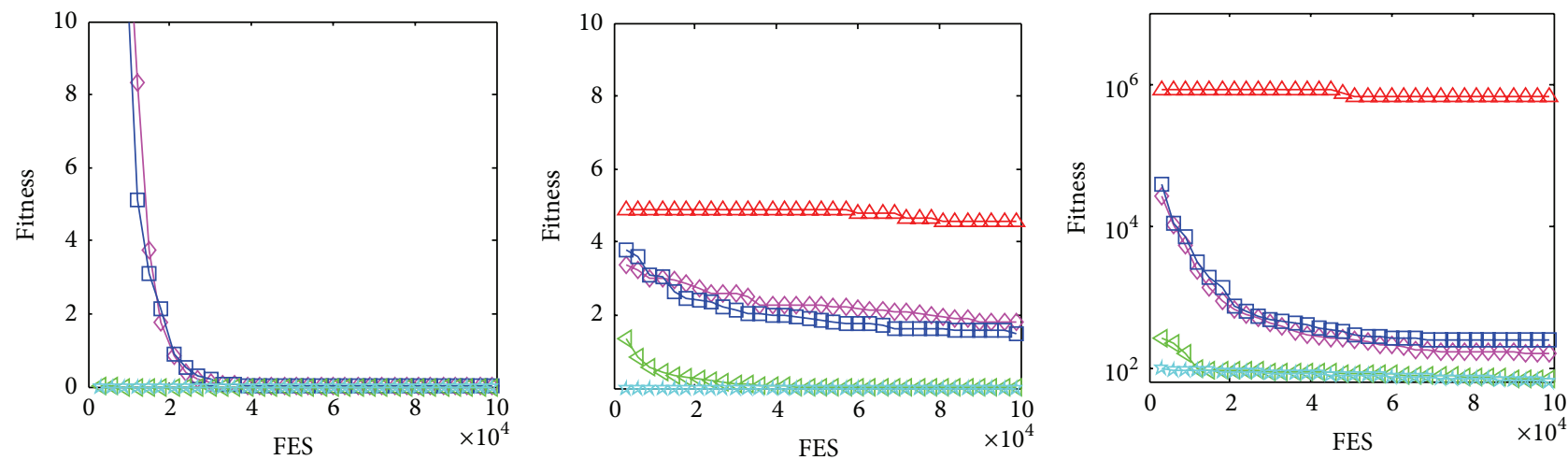

$$
\begin{array}{ll}
\triangleleft & \text { OGSO } \\
\square & \text { RGSO } \\
\triangleleft & \text { RDGSO } \\
- & \text { DGSO }
\end{array}
$$

(a) $f_{1}$

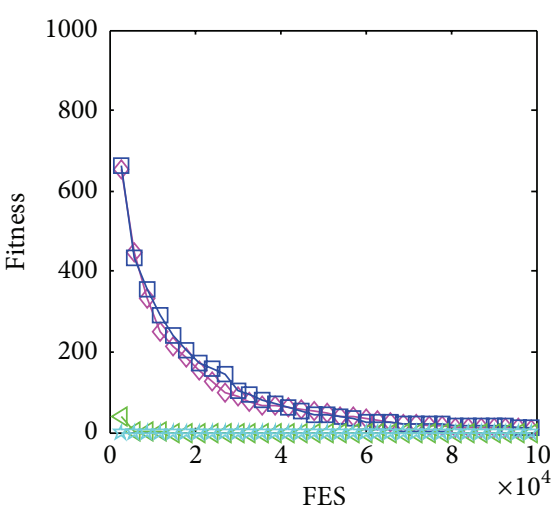

$$
\begin{aligned}
& \triangleleft \text { OGSO } \\
& \square \text { RGSO } \\
& \triangleleft \text { RDGSO } \\
& -\frac{1}{\star} \text { DGSO }
\end{aligned}
$$

(d) $f_{4}$

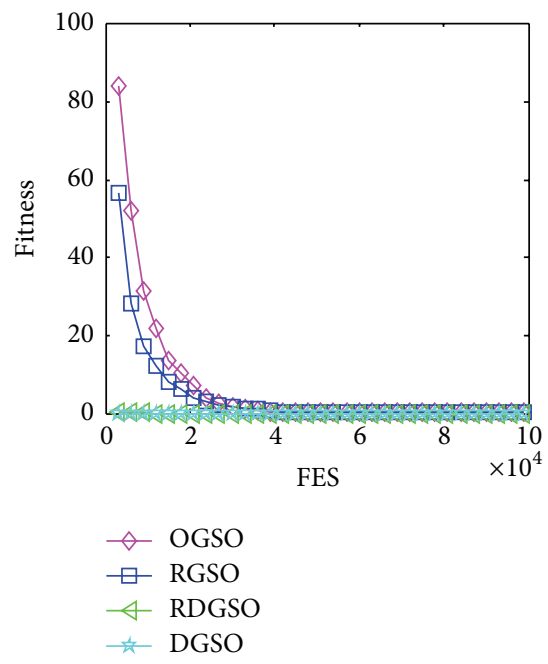

(g) $f_{7}$

$$
\begin{aligned}
& \triangle \text { GSO } \\
& \triangleleft \text { OGSO } \\
& \square \text { RGSO } \\
& \triangleleft \text { RDGSO } \\
& - \text { DGSO }
\end{aligned}
$$

(b) $f_{2}$

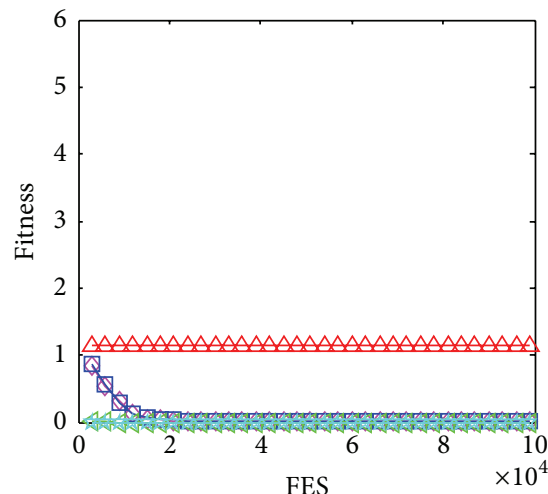

$$
\begin{aligned}
& \triangle \text { GSO } \\
& \triangleleft \text { OGSO } \\
& \square \text { RGSO } \\
& \triangleleft \text { RDGSO } \\
& \square \text { DGSO }
\end{aligned}
$$

(e) $f_{5}$

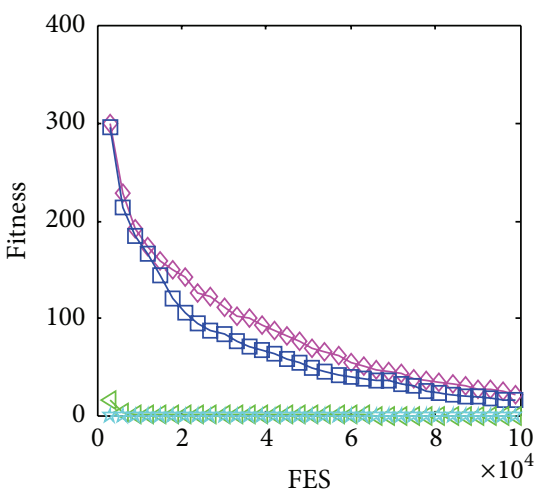

$$
\begin{aligned}
& \triangleleft \text { OGSO } \\
& \square \text { RGSO } \\
& \triangleleft \text { RDGSO } \\
& \star-\text { DGSO }
\end{aligned}
$$

(h) $f_{8}$

$$
\begin{aligned}
& \triangle \text { GSO } \\
& \triangleleft \text { OGSO } \\
& \square \text { RGSO } \\
& \triangleleft \text { RDGSO } \\
& - \text { DGSO }
\end{aligned}
$$

(c) $f_{3}$

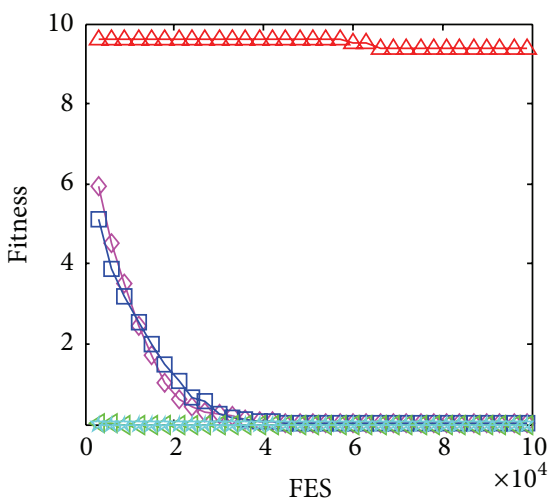

$$
\begin{aligned}
& \triangle \text { GSO } \\
& \triangleleft \text { OGSO } \\
& \square-\text { RGSO } \\
& \triangleleft \text { RDGSO } \\
& - \text { DGSO }
\end{aligned}
$$

(f) $f_{6}$

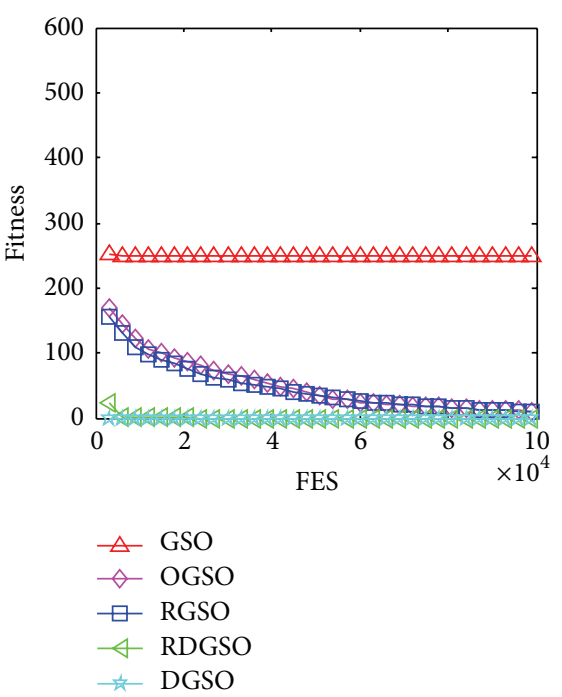

(i) $f_{9}$

FIgUre 1: Continued. 

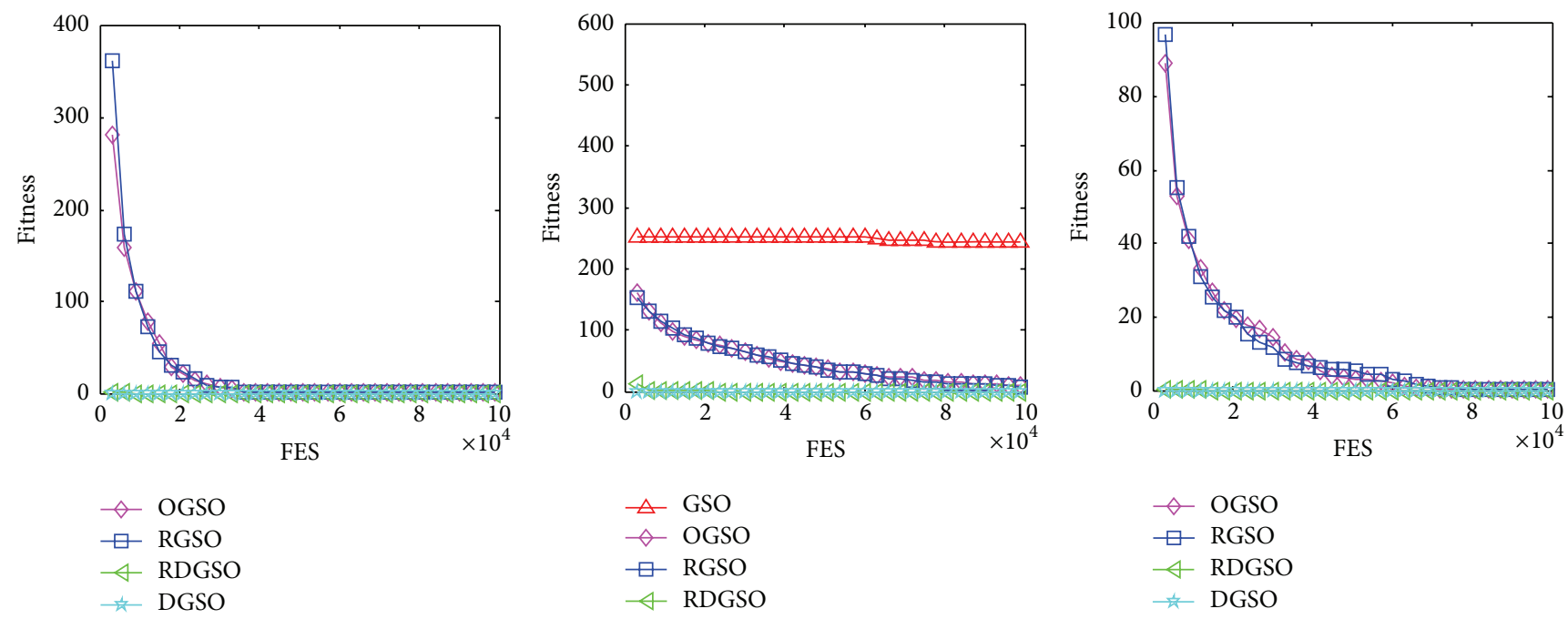

(j) $f_{10}$
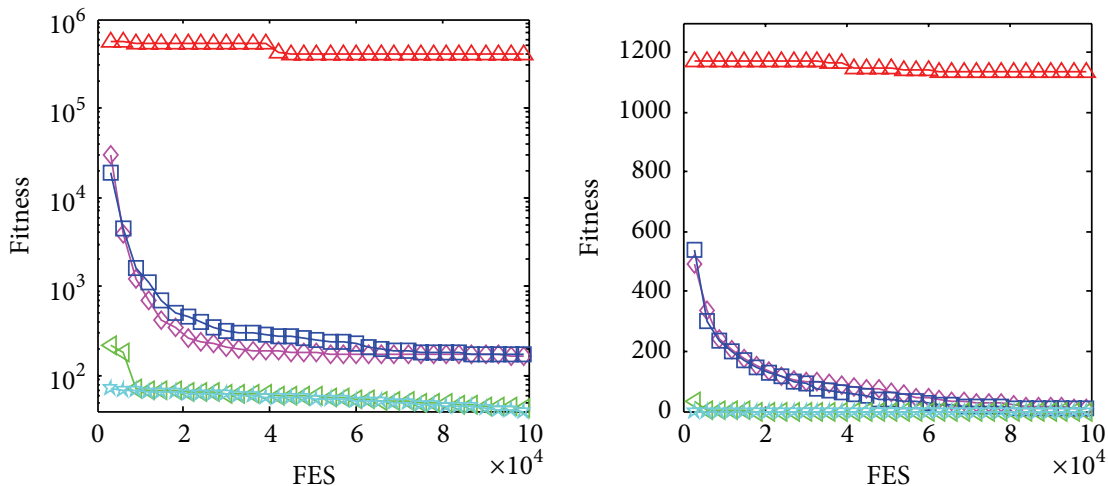

(l) $f_{12}$

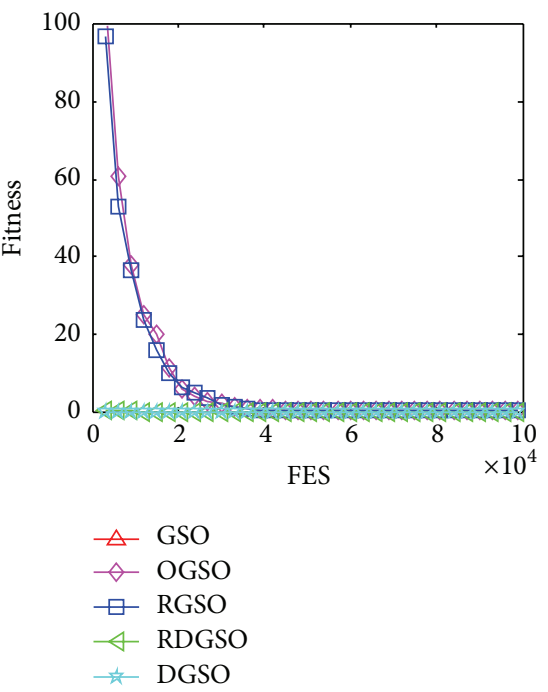

(m) $f_{13}$
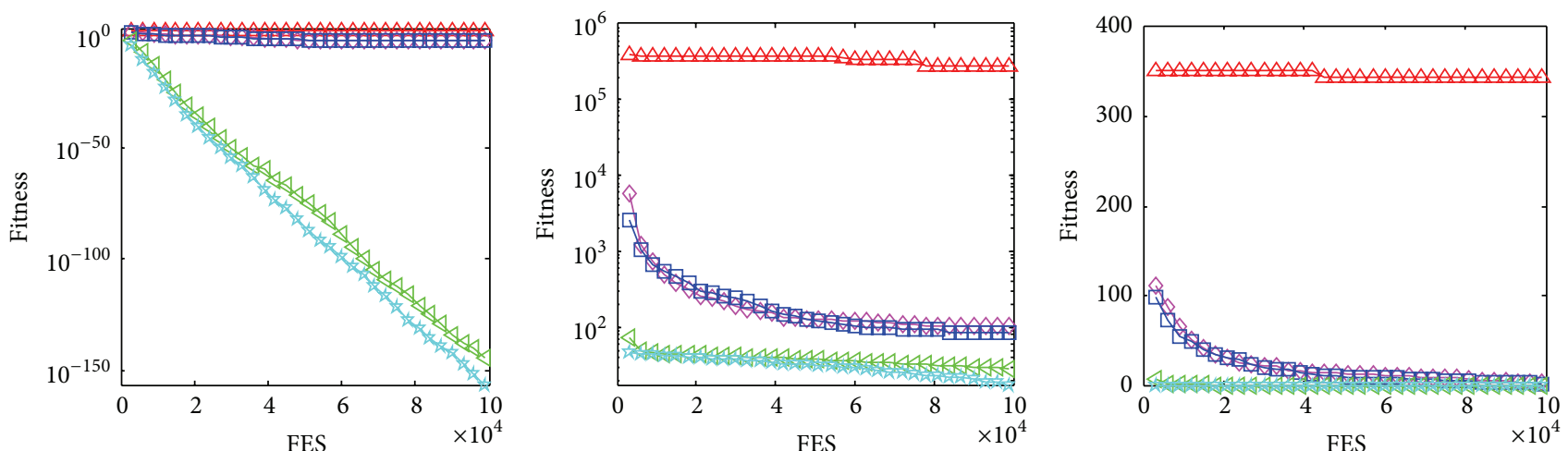

$$
\begin{aligned}
& \triangle \text { GSO } \\
& \triangleleft \text { OGSO } \\
& \square \text { RGSO } \\
& \triangleleft \text { RDGSO } \\
& \square \text { DGSO }
\end{aligned}
$$

$$
\begin{aligned}
& \triangle \text { GSO } \\
& \triangleleft \text { OGSO } \\
& \square \text { RGSO } \\
& \triangleleft \text { RDGSO } \\
& \square \text { DGSO }
\end{aligned}
$$

$$
\begin{aligned}
& \triangle \text { GSO } \\
& \triangleleft \text { OGSO } \\
& \square \text { RGSO } \\
& \triangleleft \text { RDGSO } \\
& \square \text { DGSO }
\end{aligned}
$$

\section{(p) $f_{16}$}

(r) $f_{18}$

FIgURE 1: Continued. 


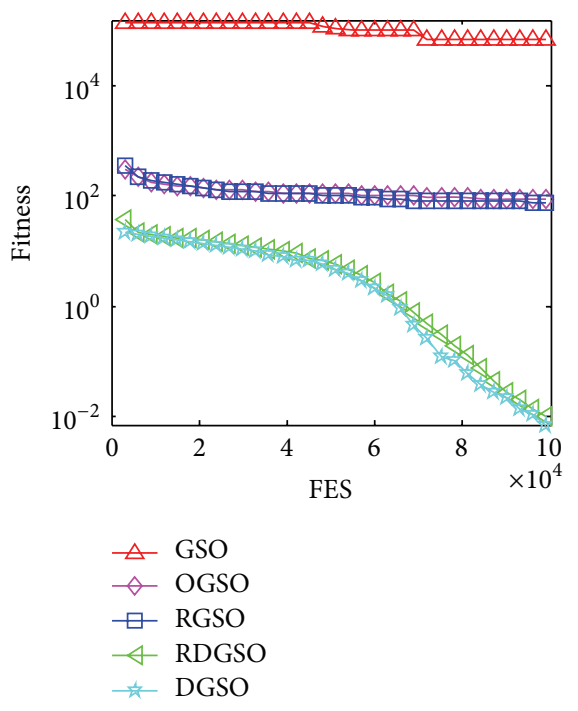

(s) $f_{19}$

FIGURE 1: Convergence results for test functions $f_{1}-f_{19}$.

\section{Acknowledgments}

This work was supported by the Foundation of Educational Commission of Tianjin City, China (Grant no. 20140803), supported by the Innovation Foundation for Young Teachers of Tianjin University of Science and Technology, China (Grant no. 2014CXLG30), supported by the National Natural Science Foundation of China (Grants nos. 61272509, 61402332, and 61402331), and supported by National High Technology Research and Development Program of China (Grant no. SQ2011SF11801036).

\section{References}

[1] F. J. Rodriguez, C. García-Martinez, and M. Lozano, "Hybrid metaheuristics based on evolutionary algorithms and simulated annealing: taxonomy, comparison, and synergy test," IEEE Transactions on Evolutionary Computation, vol. 16, no. 6, pp. 787-800, 2012.

[2] W. Huang and L. X. Ding, "Project-scheduling problem with random time-dependent activity duration times," IEEE Transactions on Engineering Management, vol. 58, no. 2, pp. 377-387, 2011.

[3] W. Huang and L. X. Ding, "The shortest path problem on a fuzzy time-dependent network," IEEE Transactions on Communications, vol. 60, no. 11, pp. 3376-3385, 2012.

[4] J.-N. Choi, S.-K. Oh, and W. Pedrycz, "Structural and parametric design of fuzzy inference systems using hierarchical fair competition-based parallel genetic algorithms and information granulation," International Journal of Approximate Reasoning, vol. 49, no. 3, pp. 631-648, 2008.

[5] R. Storn and K. Price, "Differential evolution-a simple and efficient adaptive scheme for global optimization over continuous spaces," Tech. Rep. TR-95-012, Berkeley, Calif, USA, 1995.

[6] K. V. Price, R. M. Storn, and J. A. Lampinen, Differential Evolution: A Practical Approach to Global Optimization, Springer, Berlin, Germany, 2005.
[7] M. Vasile, E. Minisci, and M. Locatelli, "An inflationary differential evolution algorithm for space trajectory optimization," IEEE Transactions on Evolutionary Computation, vol. 15, no. 2, pp. 267-281, 2011.

[8] A. M. Mora, P. García-Sánchez, J. J. Merelo, and P. A. Castillo, "Pareto-based multi-colony multi-objective ant colony optimization algorithms: an island model proposal," Soft Computing, vol. 17, no. 7, pp. 1175-1207, 2013.

[9] C.-F. Lu, C.-H. Hsu, and C.-F. Juang, "Coordinated control of flexible AC transmission system devices using an evolutionary fuzzy lead-lag controller with advanced continuous ant colony optimization," IEEE Transactions on Power Systems, vol. 28, no. 1, pp. 385-392, 2013.

[10] M. R. Khouadjia, B. Sarasola, E. Alba, L. Jourdan, and E.-G. Talbi, "A comparative study between dynamic adapted PSO and VNS for the vehicle routing problem with dynamic requests," Applied Soft Computing Journal, vol. 12, no. 4, pp. 1426-1439, 2012.

[11] Y. Marinakis and M. Marinaki, "Particle swarm optimization with expanding neighborhood topology for the permutation flowshop scheduling problem," Soft Computing, vol. 17, no. 7, pp. 1159-1173, 2013.

[12] S. He, Q. H. Wu, and J. R. Saunders, "A novel group search optimizer inspired by animal behavioural ecology," in Proceedings of the IEEE Congress on Evolutionary Computation (CEC '06), pp. 1272-1278, Vancouver, Canada, July 2006.

[13] S. He, Q. H. Wu, and J. R. Saunders, "Group search optimizer: an optimization algorithm inspired by animal searching behavior," IEEE Transactions on Evolutionary Computation, vol. 13, no. 5, pp. 973-990, 2009.

[14] K. A. de Jong, Evolutionary Computation: a Unified Approach, MIT Press, Cambridge, Mass, USA, 2006.

[15] S. Nesmachnow, H. Cancela, and E. Alba, "A parallel micro evolutionary algorithm for heterogeneous computing and grid scheduling," Applied Soft Computing, vol. 12, no. 2, pp. 626-639, 2012.

[16] Y. Mei, K. Tang, and X. Yao, "A memetic algorithm for periodic capacitated arc routing problem," IEEE Transactions on Systems, 
Man, and Cybernetics Part B: Cybernetics, vol. 41, no. 6, pp. 1654-1667, 2011.

[17] H. R. Tizhoosh, "Opposition-based learning: a new scheme for machine intelligence," in Proceedings of the International Conference on Computational Intelligence for Modelling, Control and Automation, vol. 1, pp. 695-701, Vienna, Austria, November 2005.

[18] H. R. Tizhoosh, "Reinforcement learning based on actions and opposite actions," in Proceedings of the ICGST International Conference on Artificial Intelligence and Machine Learning, Cairo, Egypt, 2005.

[19] W. Huang, S.-K. Oh, Z. Guo, and W. Pedrycz, "A space search optimization algorithm with accelerated convergence strategies," Applied Soft Computing, vol. 13, no. 12, pp. 46594675, 2013.

[20] W. Huang, S.-K. Oh, and W. Pedrycz, "Design of hybrid radial basis function neural networks (HRBFNNs) realized with the aid of hybridization of fuzzy clustering method (FCM) and polynomial neural networks (PNNs)," Neural Networks, vol. 60, pp. 166-181, 2014.

[21] C. Xiong, L. Hao, and D. Wang, "Group search optimizer with ranking-based differential operator," in Proceedings of the International Symposium on Information Technology Convergence (ISITC '15), vol. 4, pp. 3279-3285, Tianjin, China, October 2015.

[22] D. Mustard, "Numerical integration over the n-dimensional spherical shell," Mathematics of Computation, vol. 18, pp. 578589, 1964.

[23] G. M. Viswanathan, S. V. Buldyrev, S. Havlin, M. G. E. Da Luz, E. P. Raposo, and H. E. Stanley, "Optimizing the success of random searches," Nature, vol. 401, no. 6756, pp. 911-914, 1999.

[24] K. Li, "Performance analysis and evaluation of random walk algorithms on wireless networks," in Proceedings of the IEEE International Symposium on Parallel and Distributed Processing, Workshops and Phd Forum (IPDPSW '10), pp. 1-8, Atlanta, Ga, USA, April 2010.

[25] H. Ono, "Fast random walks on finite graphs and graph topological information," in Proceedings of the 2nd International Conference on Networking and Computing (ICNC '11), pp. 360363, IEEE, Osaka, Japan, December 2011.

[26] R. S. Rahnamayan, H. R. Tizhoosh, and M. M. A. Salama, "Opposition-based differential evolution," IEEE Transactions on Evolutionary Computation, vol. 12, no. 1, pp. 64-79, 2008.

[27] J. Riget and J. S. Vesterstrøm, "A diversity-guided particle swarm optimizer-the ARPSO," Tech. Rep. 2002-02, Department of Computer Science, University of Aarhus, EVALife, 2002.

[28] F. Herrera, M. Lozano, and D. Molina, "Test suite for the special issue of soft computing on scalability of evolutionary algorithms and other metaheuristics for large scale continuous optimization problems," Tech. Rep., University of Granada, Granada, Spain, 2010.

[29] K. Tang, X. Yao, P. N. Suganthan et al., "Benchmark functions for the CEC '2008 special session and competition on highdimensional real-parameter optimization," Tech. Rep., Nature Inspired Computation and Applications Laboratory, USTC, Hefei, China, 2008.

[30] F. Herrera and M. Lozano, "Workshop for evolutionary algorithms and other metaheuristics for continuous optimization problems-a scalability test," in Proceedings of the International Conference on Intelligent System Design and Applications (ISDA '09), Pisa, Italy, November-December 2009.
[31] N. Q. Huy, O. Y. Soon, L. M. Hiot, and N. Krasnogor, "Adaptive cellular memetic algorithms," Evolutionary Computation, vol. 17, no. 2, pp. 231-256, 2009.

[32] M. Vasile, E. Minisci, and M. Locatelli, "An inflationary differential evolution algorithm for space trajectory optimization," IEEE Transactions on Evolutionary Computation, vol. 15, no. 2, pp. 267-281, 2011.

[33] S. K. Goudos, K. A. Gotsis, K. Siakavara, E. E. Vafiadis, and J. N. Sahalos, "A multi-objective approach to subarrayed linear antenna arrays design based on memetic differential evolution," IEEE Transactions on Antennas and Propagation, vol. 61, no. 6, pp. 3042-3052, 2013.

[34] U. Halder, S. Das, and D. Maity, "A cluster-based differential evolution algorithm with external archive for optimization in dynamic environments," IEEE Transactions on Cybernetics, vol. 43, no. 3, pp. 881-897, 2013. 


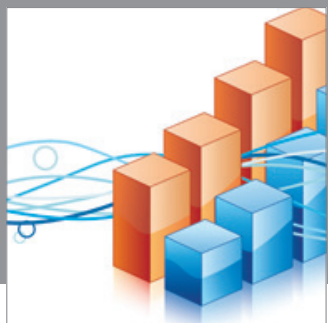

Advances in

Operations Research

mansans

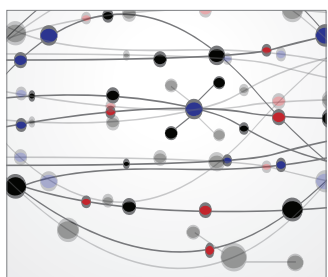

The Scientific World Journal
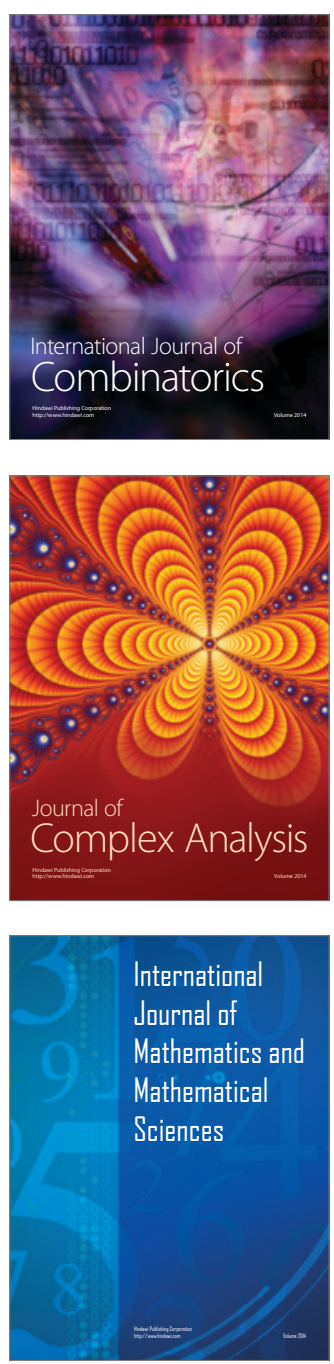
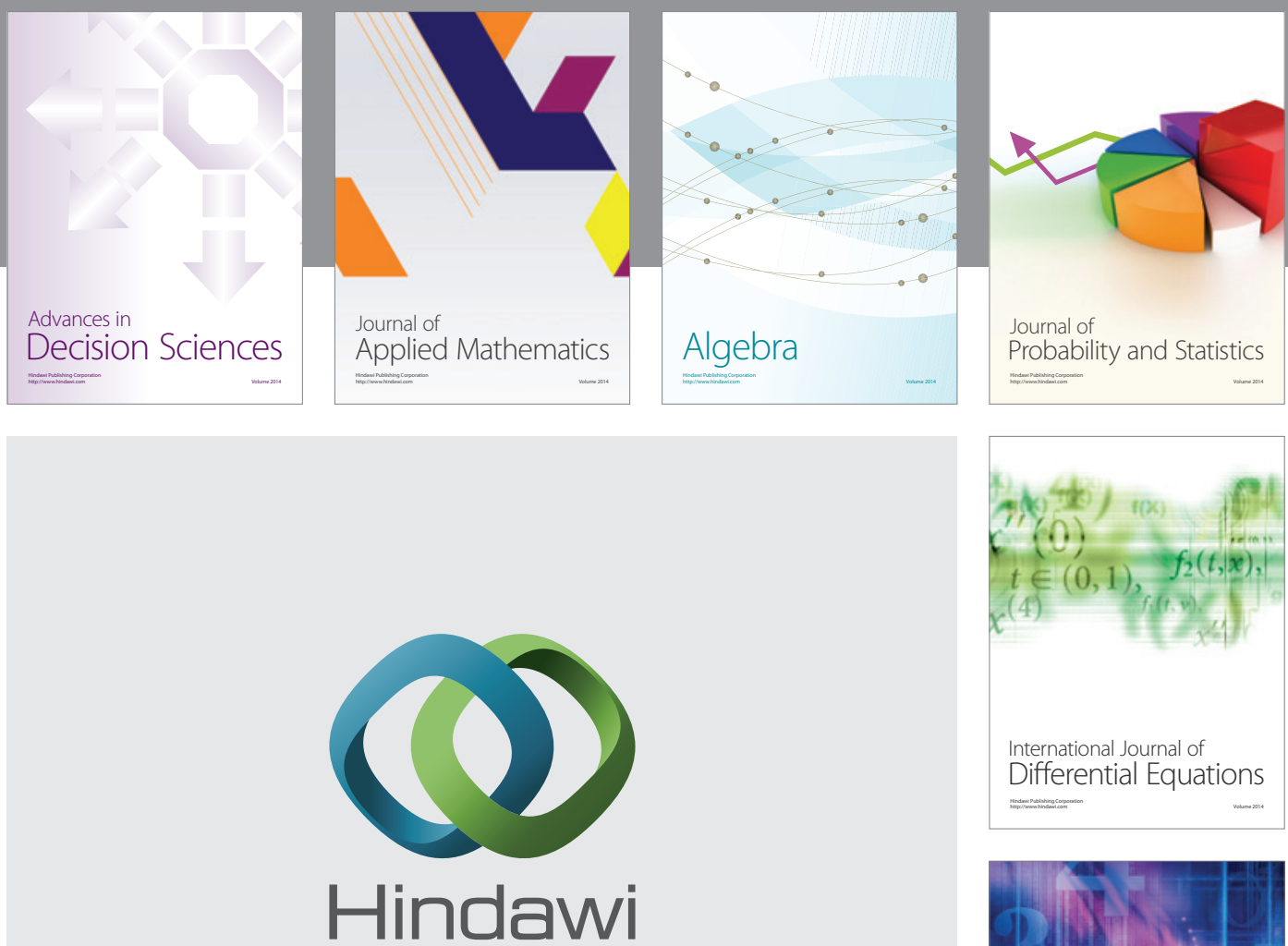

Submit your manuscripts at http://www.hindawi.com
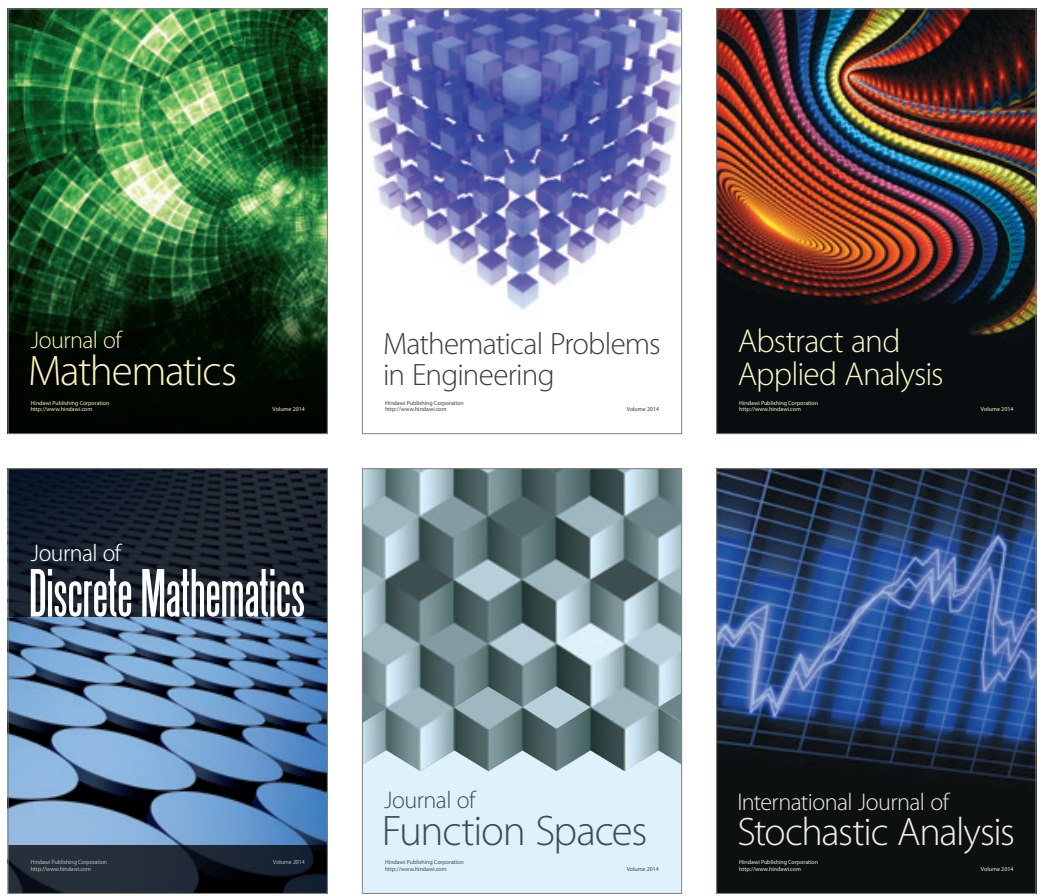

Journal of

Function Spaces

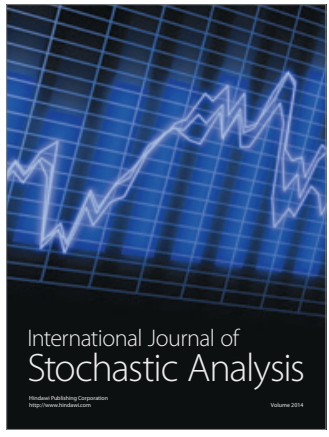

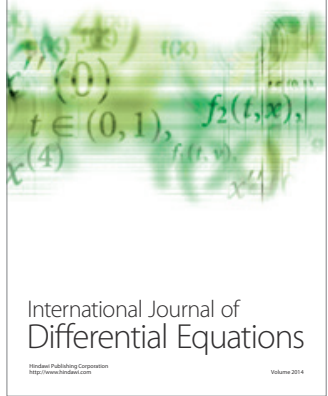
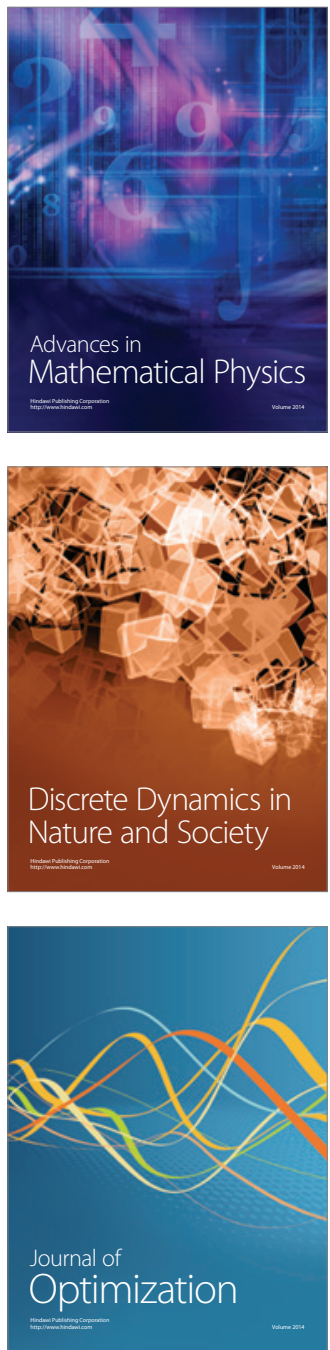